\section{FGF1 restores blood glucose levels and insulin sensitivity in diabetic mice}

A single injection of recombinant fibroblast growth factor 1 (FGF1) can normalize blood glucose levels in severely hyperglycaemic diabetic mice, according to new research published in Nature. Although preliminary, the findings suggest a possible therapeutic approach for treating metabolic diseases characterized by insulin resistance, including type 2 diabetes mellitus.

Insulin resistance leads to high blood glucose levels as a consequence of the body's failure to respond to insulin. Currently, thiazolidinediones-agonists of peroxisome proliferator-activated receptor $\gamma(\mathrm{PPAR}-\gamma)$ - are used to improve insulin sensitivity; however, their use is associated with adverse effects, such as weight gain, bone loss and heart failure. Earlier studies showing that $\mathrm{FGF1}^{-/-}$mice develop insulin resistance when fed a high-fat diet and that FGF1 is transcriptionally regulated by (and thus downstream of) PPAR- $\gamma$ suggested that
FGF1 might have glucose-lowering activity but with reduced adverse effects.

To investigate this possibility, the researchers used genetically induced obese $(o b / o b$ and $d b / d b)$ and dietinduced obese (DIO) insulin-resistant mice. A single dose of FGF1 restored normoglycaemia in all three mouse models; glucose lowering peaked within $18-24 \mathrm{~h}$ and was sustained for $48 \mathrm{~h}$. Serial doses of FGF1 delivered every other day for 35 days resulted in sustained glucose lowering without evidence of the adverse effects associated with thiazolidinedione use. Chronic treatment with FGF1 resulted in insulin sensitization, evidenced by suppression of hepatic glucose production and increased insulin-dependent glucose uptake in skeletal muscle.

A potential concern with using FGF1 therapeutically is the possible induction of unwanted cell proliferation. To address this concern, the researchers generated an FGF1 variant lacking the first 24 amino

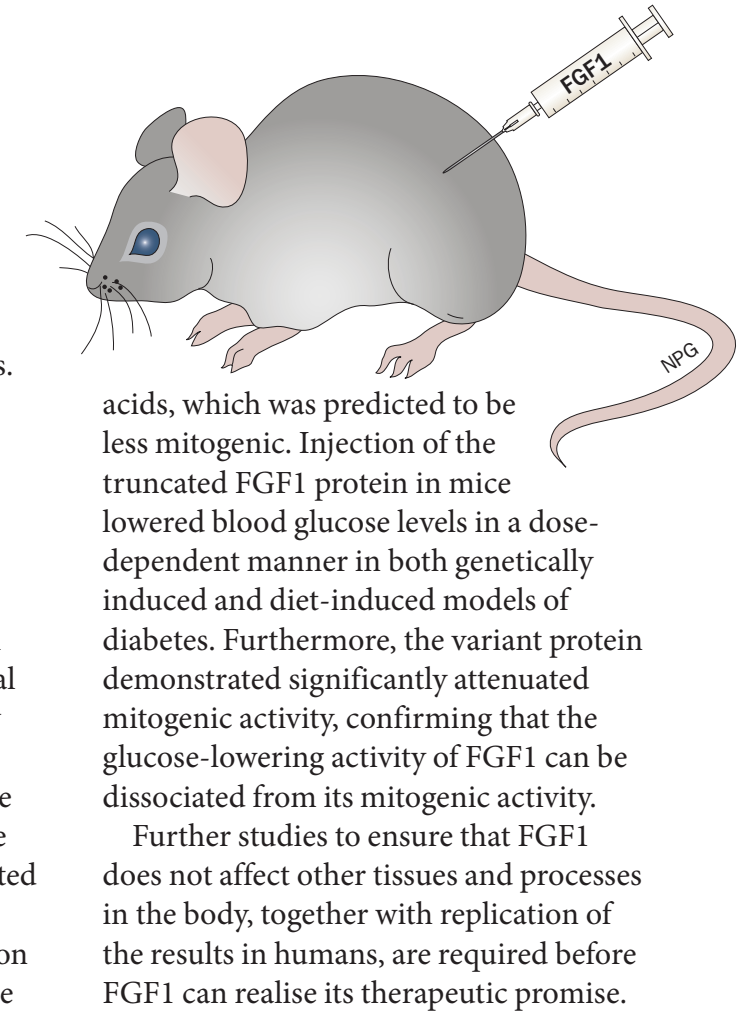

David Holmes

Original article Suh, J. M. et al. Endocrinization of FGF1 produces a neomorphic and potent insulin sensitizer. Nature doi:10.1038/nature13540 